Case Report

\title{
Intracerebral Hemorrhages Secondary to Reversible Cerebral Vasoconstriction Syndrome: Case Report and Literature Review
}

\author{
François Dantas ${ }^{1,2, ~ *}$, Eustáquio Claret dos Santos Júnior ${ }^{1,2}$, Tiago Silva e Carvalho ${ }^{1}$, \\ Róberti Uili Rodrigues Firmino ${ }^{1}$, Rômulo Tscherbakowski Nunes de Guimarães Mourão, ${ }^{1,}$, \\ Mariana Couy Fonseca ${ }^{1}$, Fernando Luiz Rolemberg Dantas ${ }^{1,3}$, Ricardo Vieira Botelho ${ }^{3}$, \\ Rogério Zenóbio Darwich ${ }^{1}$ \\ ${ }^{1}$ Neurology and Interventionist Neuroradiology Department, Biocor Institute, Minas Gerais, Brazil \\ ${ }^{2}$ Faculdade de Ciências Médicas de Minas Gerais (FELUMA), Belo Horizonte, Minas Gerais, Brazil \\ ${ }^{3}$ Postgraduation in Health Sciences, Instituto de Assistência Médica ao Servidor Público Estadual (IAMSPE), São Paulo, Brazil
}

Email address:

francois_dantas@hotmail.com(F. Dantas)

${ }^{*}$ Corresponding author

\section{To cite this article:}

François Dantas, Eustáquio Claret dos Santos Júnior, Tiago Silva e Carvalho, Róberti Uili Rodrigues Firmino, Rômulo Tscherbakowski Nunes de Guimarães Mourão, Mariana Couy Fonseca, Fernando Luiz Rolemberg Dantas, Ricardo Vieira Botelho, Rogério Zenóbio Darwich. Intracerebral Hemorrhages Secondary to Reversible Cerebral Vasoconstriction Syndrome: Case Report and Literature Review. Clinical Neurology and Neuroscience. Vol. 3, No. 3, 2019, pp. 69-73. doi: 10.11648/j.cnn.20190303.13

Received: June 10, 2019; Accepted: July 22, 2019; Published: August 12, 2019

\begin{abstract}
Reversible cerebral vasoconstriction syndrome is an unusual entity, characterized by recurrent thunderclap headache and segmental narrowing of the cerebral arteries, typically with remission within three months. It has been described since the 1960s with several names, including Call-Fleming syndrome. More than 500 cases have been described in the literature, yet the pathophysiology remains not well understood. Ischemic or hemorrhagic strokes are the major possible complications of the syndrome, leading to permanent neurological deficits or death in a small percentage of patients. We report a case of a 48-year-old female patient without known risk factors that presented two foci of intracerebral hemorrhages, with hemiparesis ipsilateral to the biggest intracerebral lesion. Magnetic resonance imaging tractography revealed normal pyramidal decussation, and the patient evolved with completely recover of the neurological deficit within a week. The authors believe that in the present case neurological deficit may be related to contralateral narrowing of the cerebral arteries and diffuse impairment of the central nervous system instead of intracerebral hemorrhage itself. RCVS is a rare condition that should be considered in patients with recurrent thunderclap type headache. Further prospective and randomized studies are still necessary to improve the management and treatment of patients with the syndrome.
\end{abstract}

Keywords: Reversible Cerebral Vasoconstriction, Intracerebral Hemorrhages, Thunderclap Headache

\section{Introduction}

Reversible cerebral vasoconstriction syndrome (RCVS) is an unusual entity, which features recurrent crisis of thunderclap headache associated with diffuse segmental narrowing of cerebral arteries and, in most cases, spontaneous resolution of the angiographic alterations within three months.

The increase in the number of diagnoses observed recently may be related to a higher level of suspicion and improvement of the imaging techniques. More than 500 cases have been described in the literature, with one third of patients evolving with cerebral ischemia, intracranial hemorrhage or both, and only $5-10 \%$ with permanent deficits or death [1].

We report a case of a patient with RCVS that presented two foci of intracerebral hemorrhage besides bilateral subarachnoid hemorrhage, with neurological deficit ipsilateral 
to the biggest intracerebral lesion.

\section{Case Report}

A 48-year-old woman, with regular use of Enalapril due to a history of hypertension, without other illnesses or known toxic substance abuse, presented with three episodes of thunderclap headache over a week on March 2018, with complete and spontaneous resolution of the pain in a few hours. She sought emergency care on the second episode, being medicated with common analgesics and discharged.

She attended our service during the third episode of headache, which coursed with neurological deficit. On admission, her blood pressure was 210 x $120 \mathrm{mmHg}$ and heart rate $104 \mathrm{bpm}$. The neurological examination revealed hemiparesis of the right dimidium, grade 3 (Medical Research Council Scale for Muscle Strength) and Babinski's sign on the same side. The rest of the physical and neurological examination was normal.

Computed Tomography scan (CT scan) of the skull showed two foci of intracerebral hemorrhage, one located at the temporo-occipital region, the other at the frontal lobe, both on the right hemisphere, with mild edema around, besides subarachnoid (intergyral) hemorrhage in the left parietal convexity (Figure 1). Brain Magnetic Resonance Image (MRI) showed subarachnoid hemorrhage in the right Sylvian fissure and both convexities of the brain (Figure 2). Magnetic Resonance Angiography (MRA) showed multiple spots of segmental vasoconstriction in the right middle cerebral artery
(Figure 3), without evidence of aneurysms. The venous phase of the MRA was normal.

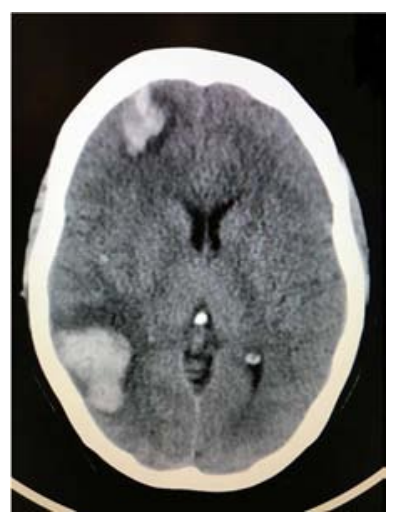

Figure 1. CT Scan showing two foci of intracerebral hemorrhages, one at the temporo-occiptal region and another at the frontal lobe.

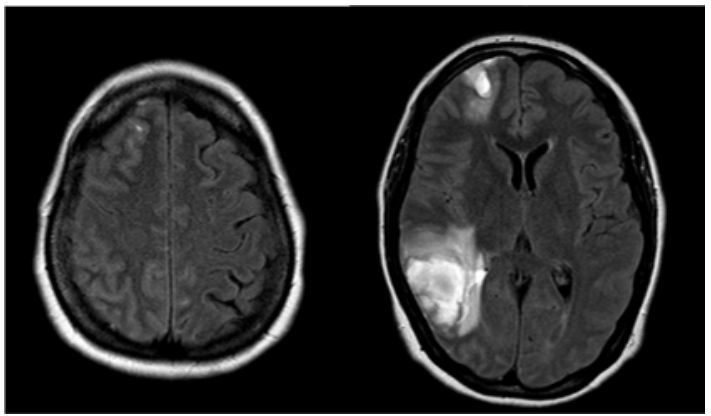

Figure 2. MRI demonstrating intracerebral hemorrhages and convexity subarachnoid hemorrhage.

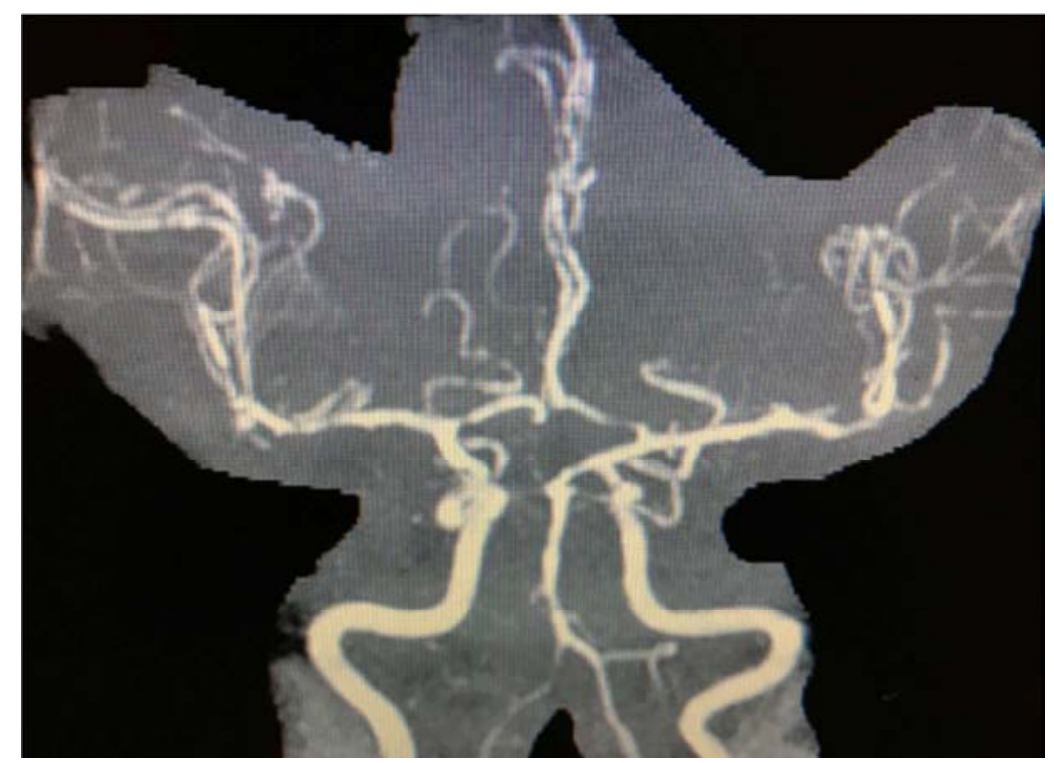

Figure 3. MRA. Multiple spots of segmental vasoconstriction in the right middle cerebral artery.

Patient was admitted to the Intensive Care Unit, where pressure control was performed with Nitroprussiate, and Nimodipine $60 \mathrm{mg}$ was administered orally every four hours. Afterwards she did not present with new episodes of thunderclap headache, rather evolving with progressive improvement of neurological deficit and complete resolution of symptoms within a week. She was submitted to cervical and cerebral Digital Subtraction Angiography (DSA), confirming multiple focal areas of vasoconstriction in the anterior and posterior circulation (Figure 4). MRI tractography was also performed to invesigate neurological deficit ipsilateral to the intracerebral hemorrhage spots. Imaging showed usual decussation of motor fibers at the level of the bulbar pyramids (Figure 5). 

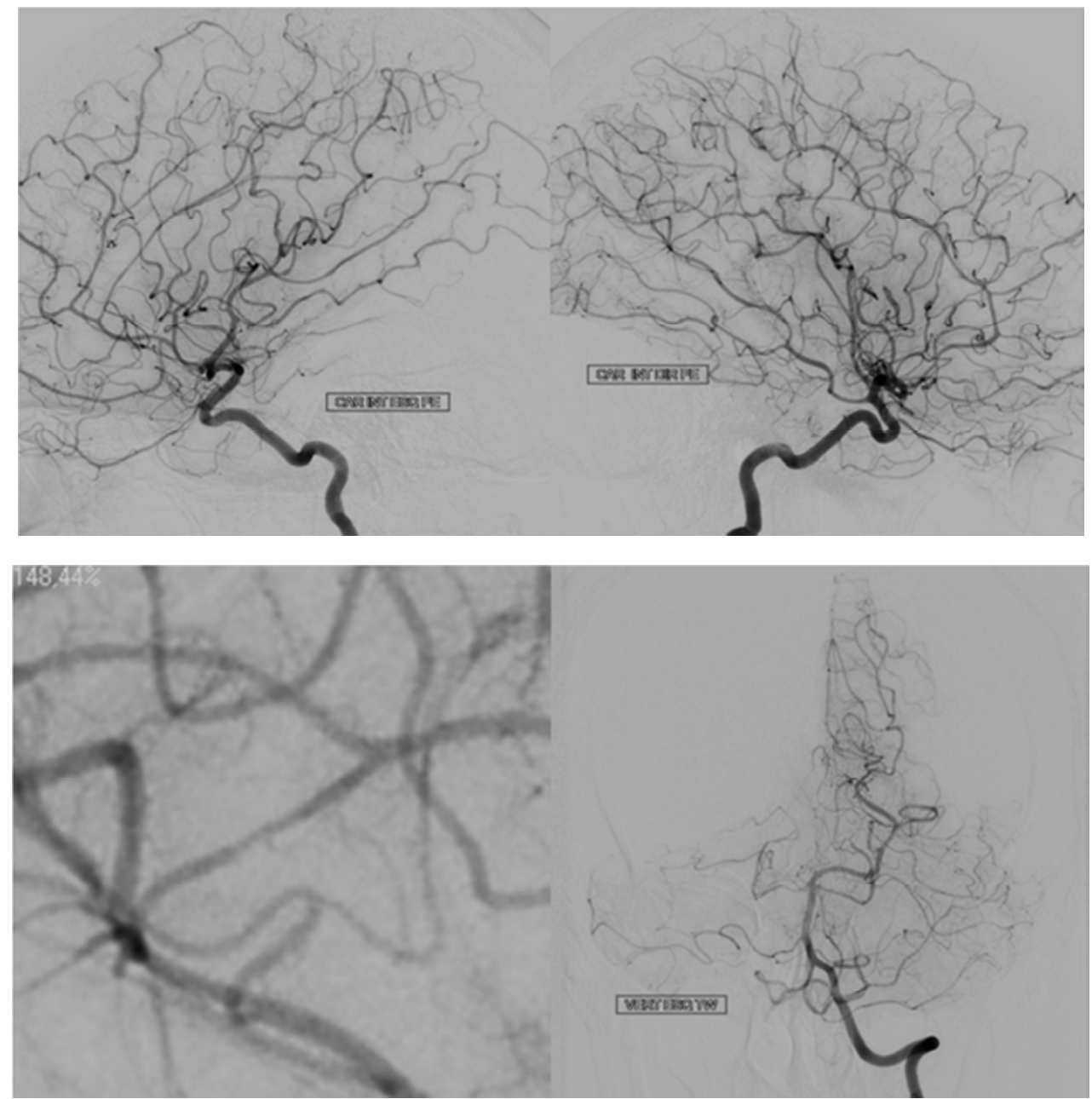

Figure 4. DSA with multiple focal stenosis in the anterior $(A, B, C)$ and posterior (D) circulation, confirming the diagnosis of RCVS.

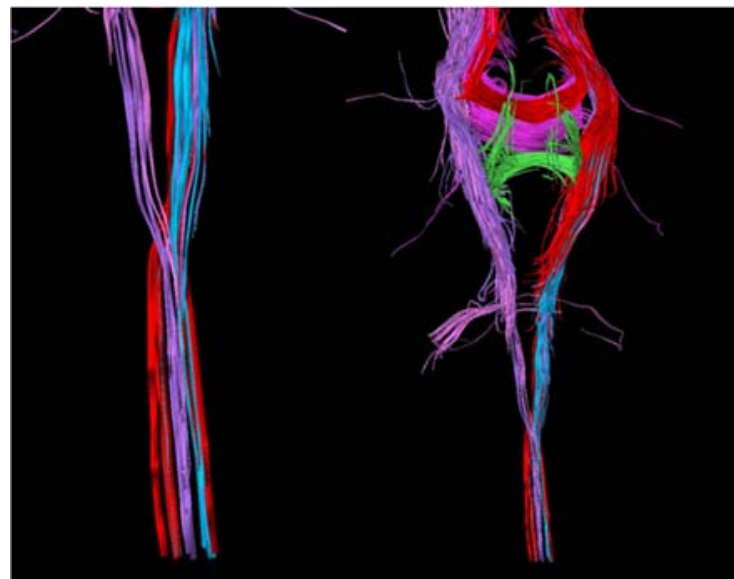

Figure 5. MRI tractography. Habitual pyramidal decussation.

Several secondary causes of hypertension were investigated including pheochromocytoma and rheumatologic diseases, none were confirmed. The patient was discharged asymptomatic and without deficits, with no recurrence of headache or elevate pressure levels in subsequent outpatient follow-up.

\section{Discussion}

Reversible cerebral vasoconstriction syndrome (RCVS) is characterized mainly by recurrent attacks of sudden and intense thunderclap headache, with or without acute neurological deficits, and the presence of diffuse and segmental constriction of cerebral arteries, which are resolved spontaneously within three months. Headache is usually the first symptom and is typically recurrent within the first two weeks. Ischemic or hemorrhagic stroke are the main complications of the syndrome [2].

The syndrome has been described since the 1960s with several names, including Call-Fleming syndrome, postpartum angiopathy, migrainous vasospasm, benign angiopathy of the CNS, benign cerebral vasculitis, CNS pseudovasculitis and reversible cerebral segmental vasoconstriction [2-4]. There are case reports of patients aged 9 to 76 years, but the syndrome typically occurs between 20-50 years (mean age of 42-45 years) and is more prevalent in women (2: 1 to $10: 1$, varying among the series in the literature) $[1,3]$.

The diagnostic criteria for the syndrome were proposed by Calabrese et al in 2007 [5] and modified by the International 
Headache Society [6]. They have not been validated in prospective studies, but they have good precision in the diagnosis and knowledge of the pathology [3]. They include: (1) Severe and sudden headache with or without focal deficits or seizures; (2) Uniphasic course without new symptoms more than one month after clinical onset; (3) Segmental vasoconstriction of cerebral arteries evidenced by DSA, MRA or Computed Tomography Angiography (CTA) (4) Absence of evidence of aneurysmal subarachnoid hemorrhage; (5) Normal or near-normal rates of protein in the cerebrospinal fluid $(<80 \mathrm{mg} / \mathrm{dL})$; (6) Complete normalization of arteries shown by imaging examinations within 12 weeks after symptoms onset.

RCVS can occur spontaneously, with no identifiable cause, or may be secondary to triggers, observed in $25-60 \%$ of cases $[7,8]$, mainly the use of vasoactive drugs and puerperium, which together correspond to more than half the cases published (approximately 50 and $10 \%$ of cases, respectively). It is commonly associated with migraine (20-40\% of cases) and sometimes related to arterial dissection, although it is not yet possible to state whether dissection is a complication or cause of the disease. Some case series relate RCVS and cannabis use [3].

Other causes include catecholamine-secreting tumors such as pheochromocytoma, use of human immunoglobulin or interferon alfa, red blood cell transfusion, sexual activity, Valsalva maneuver, hypercalcemia, traumatic brain injury, phenytoin intoxication, cerebrospinal fluid hypotension, neurosurgery, among others [2, 3]. Even airplane travel and herbal supplements have been described as triggers for the syndrome $[9,10]$.

Pathophysiology is still unknown, but it is believed that changes in brain vessel tone leading to vasoconstriction are responsible for the development of the syndrome, and may be induced by sympathetic hyperactivity, endothelial dysfunction and oxidative stress. The overlap between RCVR and Posterior Reversible Encephalopathy Syndrome (PRES) suggests an important role of endothelial dysfunction in the disease $[2,3,11]$.

The RCVR headache is usually thunderclap type, that is, intense, with pain reaching its peak within 1 minute, and affects $94-100 \%$ of patients. It is usually bilateral and may be associated with nausea, vomiting, diplopia, photophobia, and hypertensive peaks. Unlike other causes of thunderclap headache (non-traumatic subarachnoid hemorrhage, pituitary apoplexy, cerebral venous thrombosis, cervical arterial dissection, among others), RCVR headache is usually resolved within three hours, reoccurring one to four weeks later, with an average of four episodes of pain until complete resolution of symptoms $[2,3]$.

Laboratory tests such as blood count, coagulation parameters, electrolytes, renal and hepatic function are normal in most patients, as well as rheumatologic tests, including rheumatoid factor, ANA and Anca. Pheochromocytoma screening is indicated for all patients as well as toxicological tests to exclude drug use. Cerebrospinal fluid (CSF) abnormalities were observed in $0-60 \%$ of patients, and included an increase in cellularity, red blood cells and proteins [2].

Brain lesions are seen in neuroimaging in $12-81 \%$ of patients and include convexity subarachnoid hemorrhage, intracerebral hemorrhage, ischemia and reversible cerebral edema. Singhal et al studied 139 patients, of whom $81 \%$ presented alterations in the imaging exams, $34 \%$ of them with subarachnoid hemorrhage, $20 \%$ with intracerebral hemorrhage, $39 \%$ with ischemia and $38 \%$ with PRES [12].

Treatment and management of RCVS are based on observational studies and expert opinion. There are no randomized clinical trials for treatment of the syndrome, but early recognition is important for appropriate treatment and propaedeutics. All patients should receive analgesics and antiemetic drugs in case of nausea or vomiting, and precipitating or aggravating factors of the disease should be identified and eliminated. In addition to these medications, treatment also includes the use of antiepileptic drugs for patients with seizures, blood pressure monitoring, and ICU admission. Drugs for vasospasm can be considered, such as Nimodipine, Verapamil and Magnesium Sulphate, and can be used for up to 12 weeks. Studies have shown that Nimodipine tends to reduce the number and intensity of headaches, but does not alter the timecourse of cerebral vasoconstriction. Glucocorticoids should be avoided as they do not prevent clinical deterioration and have been associated with worsening of clinical outcome [2]. In cases of severe vasoconstriction, endovascular treatment with the use of intra-arterial vasodilators or balloon angioplasty of larger vessels has been described [13-16].

The prognosis is good in most patients with improvement of headache and angiographic pattern within days to weeks. Long-term prognosis depends on the presence or absence of stroke associated with the syndrome. Less than $10 \%$ of patients develop permanent deficits and less than $2 \%$ die [1].

\section{Conclusion}

We report a case of a patient with RCVS who had two distinct intracerebral hemorrhages and bilateral subarachnoid hemorrhages in convexities with neurological deficit in the same side of the intracerebral lesions. We believe that in the present case neurological deficit may be related to contralateral narrowing of cerebral arteries instead of intracerebral hemorrhage itself.

RCVS is a rare condition that should be considered in patients with recurrent thunderclap type headache. It can affect patients of all ages, with a predominance in females. It appears to be the main isolated cause of recurrent thunderclap type headache and should be considered as a possible cause of ischemic and hemorrhagic stroke. Multicentric prospective studies are still needed to assist with the accurate management of patients with the syndrome.

\section{Conflict of Interest}

The authors declare that there is no conflict of interest 
regarding the publication of this article.

\section{References}

[1] Cappelen-Smith C, Calic Z, Cordato D. Reversible Cerebral Vasoconstriction Syndrome: Recognition and Treatment. Curr Treat Options Neurol 2017. 19: 21.

[2] Ducross A. Reversible cerebral vasoconstriction syndrome. Lancet Neurol 2012. 11: 906-17.

[3] Miller TR, Shivashankar M, Mossa-Basha M, Gandhi D. Reversible Cerebral Vasoconstriction Syndrome, Part 1: Epidemiology, Pathogenesis and Clinical Course. AJNR Am J Neuroradiol 2015. 36: 1392-99.

[4] Call GK, Fleming MC, Sealfon S, Levine H, Kistler JF, Fisher CM. Reversible Cerebral Segmental Vasoconstriction. Stroke 1988. 19: 1159-70.

[5] Calabrese LH, Dodick DW, Schwedt TJ, Singhal AB. Narrative review: Reversible cerebral vasoconstriction syndromes. Ann InternMed 2007. 146: 34-44.

[6] Headache classification subcommittee of the International Headache Society. The international classification of headache disorders. Cephalalgia 2004. 24: 1-160.

[7] Ducros A, Bousser MG. Reversible cerebral vasoconstriction syndrome. Pract Neurol 2009. 9: 256-67.

[8] Gupta S, Zivadinov R, Ramasamy D, et al. Reversible cerebral vaso constriction syndrome (RCVS) in antiphospholipid antibody syndrome (APLA): the role of centrally acting vasodilators-case series and review of literature. Clin Rheumatol 2014; 33: 1829-33.
[9] Hiraga A, Aotsuka Y, Koide K, Suwabara. Reversible cerebral vasoconstriction syndrome precipitated by airplane descent: Case report. Cephalalgia 2016. 0 (0) 1-4.

[10] Costa I, Mendonça MD, Cruz e Silva V, Calado S, Viana-Baptista M. Herbal Supplements Association with Reversible Cerebral Vasoconstriction Syndrome: A Case Report. Journal of Stroke and Cerebrovascular Diseases 2017. Mar; 26 (3): 673-676.

[11] Choi HA, Lee MJ, Chung CS. Cerebral endothelial dysfunction in reversible cerebral vasoconstriction syndrome: a case-control study. The Journal of Headache and Pain 2017. 18: 29.

[12] Singhal AB, Hajj-Ali RA, Topcuoglu MA, et al. Reversible cerebral vasoconstriction syndromes: analysis of 139 cases. Arch Neurol 2011. 68: 1005-12.

[13] Song JK, Fisher S, Seifert TD, et al. Postpartum cerebral angiopathy: atypical features and treatment with intracranial balloon angioplasty. Neuroradiology 2004; 46: 1022-26.

[14] Elstner M, Linn J, Muller-Schunk S, Straube A. Reversible cerebral vasoconstriction syndrome: a complicated clinical course treated with intra-arterial application of nimodipine. Cephalalgia 2009; 29: 677-82.

[15] Linn J, Fesl G, Ottomeyer C, et al. Intra-arterial application of nimodipine in reversible cerebral vasoconstriction syndrome: a diagnostic tool in select cases? Cephalalgia 2011;31: 1074-81.

[16] Santos L, Azevedo E. Reversible cerebral vasoconstriction syndrome - A narrative revision of the literature. Porto Biomed. $J$ 2016; 1 (2): 65-71. 